\section{An easy, low-cost method for achieving adequate facial hair coverage for surgeons}

\section{Saqib Masud, John Glynne Andrew}

Department of Trauma and Orthopaedics, Gwynedd Hospital, Penrhosgarnedd, Bangor, UK

\section{CORRESPONDENCE TO}

Saqib Masud, E: saqib_masud@hotmail.com

\section{BACKGROUND}

Intra-operative wound contamination can have potentially disastrous consequences in orthopaedic surgery. ${ }^{1}$ Surgeons with large facial hair may be at increased risk of causing inadvertent wound contamination unless the facial hair is adequately covered. Surgical hoods are designed to achieve this but not all departments provide them due to their cost ( $£ 50.89$ for box of 40; Mölnlycke Health Care, Irlam, Manchester M44 5BJ, UK). We describe an alternative, and cheaper, method of achieving adequate facial hair coverage with items readily available in most surgical theatres.

\section{TECHNIQUE}

The surgeon should first wear an 'All' type of theatre hat ( $£ 19.28$ for box of 100; Mölnlycke Health Care) to ensure coverage of the hair and sideburns (Fig. 1). Then, taking a standard surgical mask (£3.81 for box of 60; Mölnlycke Health Care), he should place the upper-most border under his bottom lip and secure it provisionally in place by tying the upper-most ties. He should then proceed to tuck
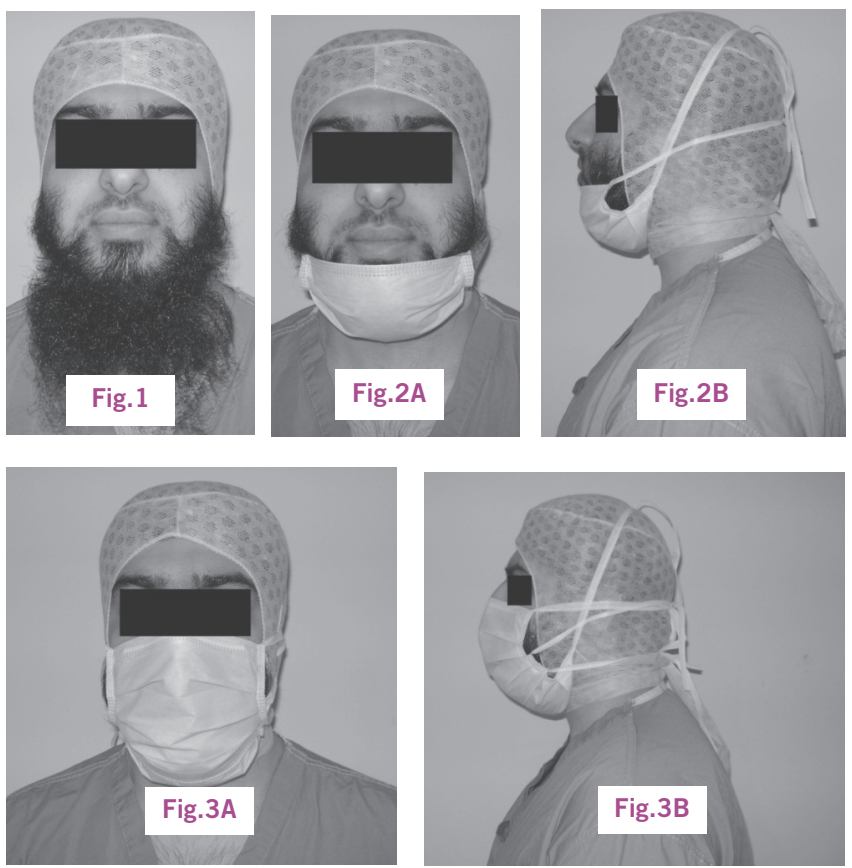

Figure 1 Appearance after wearing theatre hat.

Figure 2 Appearance from (A) front and (B) side after wearing first mask. Figure 3 Final appearance from $(A)$ front and $(B)$ side after wearing both masks; showing almost complete coverage of facial hair. his entire beard into the mask, and secure the remaining pair of ties on the top of his head (Fig. 2). The precautions can be completed with a second face mask applied in the usual manner (Fig. 3).

\section{DISCUSSION}

This simple alternative provides adequate coverage of facial hair and can be achieved using items available in most theatre departments. Despite the use of an additional face mask, the cost still compares favourably with the use of a surgical hood and single mask (32p versus $£ 1.34$ ).

\section{Reference}

1. Byrne AM, Morris S, McCarthy T, Quinlan W, O'Byrne JM. Outcome following deep wound contamination in cemented arthroplasty. Int Orthop 2007; 31: 27-31.

\section{A simple method to prevent spectacle lenses misting up on wearing a face mask}

\section{Sheraz Shafi Malik ${ }^{1}$, Shahbaz Shafi Malik ${ }^{2}$}

${ }^{1}$ Department of Trauma and Orthopaedics, Bedford Hospital NHS Trust, Bedford, UK

${ }^{2}$ Trauma and Orthopaedics (ST1), Heart of England NHS Foundation Trust, Birmingham, UK

\section{CORRESPONDENCE TO}

Sheraz Shafi Malik, E: smalik888@gmail.com

\section{BACKGROUND}

Theatre staff who wear spectacles can find their spectacle lenses misting up on wearing a face mask. This effect can be a nuisance and even incapacitate the person. A simple method to prevent this annoying phenomenon is highlighted.

\section{TECHNIQUE}

Immediately before wearing a face mask, wash the spectacles with soapy water and shake off the excess. Then, let the spectacles air dry or gently dry off the lenses with a soft tissue before putting them back on. Now the spectacle lenses should not mist up when the face mask is worn.

\section{DISCUSSION}

The face mask directs much of the exhaled air upwards where it gets into contact with the spectacle lenses. The misting occurs from the warm water vapour content condensing on the cooler surface of the lens, and forming tiny droplets that scatter the light and reduce the ability of the lens to transmit contrast. 1 The droplets form because of the inherent surface tension between the water molecules. Washing the spectacles with soapy water leaves behind a thin surfactant film that reduces this surface tension and causes the water molecules to spread out evenly into a transparent layer. This 'surfactant effect' is widely utilised to prevent misting of surfaces in many everyday situations.

\section{Reference}

1. Margrain TH, Owen C. The misting characteristics of spectacle lenses. Ophthalmic Physiol Opt 1996; 16: 108-14. 